Jodra, P. y Domínguez, R. (2020) Effects of Physical Activity on the Perceived General Health of Teachers. Revista Internacional de Medicina y Ciencias de la Actividad Física y el Deporte vol. 20 (77) pp. 155-166 Http://cdeporte.rediris.es/revista/revista77/artefectos1109.htm

DOI: $10.15366 /$ rimcafd2020.77.010

\title{
ORIGINAL
}

\section{EFECTOS DE LA ACTIVIDAD FÍSICA EN LA SALUD GENERAL PERCIBIDA DE DOCENTES}

\section{EFFECTS OF PHYSICAL ACTIVITY ON THE PERCEIVED GENERAL HEALTH OF TEACHERS}

\author{
Jodra, P. ${ }^{1,2}$ y Domínguez, . $^{3}$ \\ ${ }^{1}$ Departamento de Ciencias de la Educación, Universidad de Alcalá de Henares \\ (España) pablo.jodraj@uah.es \\ ${ }^{2}$ Departamento de Ciencias de la Actividad Física y el Deporte, Universidad Alfonso X El Sabio \\ (España) pjodriim@uax.es \\ ${ }^{3}$ Facultad de Ciencias de la Salud, Universidad Isabel I (España) raul.dominguez@ui1.es
}

Código UNESCO / UNESCO code: 6306.05 Sociología de la Educación / Educational Sociology

Clasificación Consejo de Europa / Council of Europe Classification: 16. Sociología del Deporte / Sociology of Sport

Recibido 12 de marzo de 2018 Received March 12, 2018

Aceptado 14 de julio de 2019 Accepted July 14, 2019

\section{RESUMEN}

La función docente conlleva un desgaste físico y emocional que repercute en el estado de salud de las personas. Este estudio pretende evaluar el efecto ser físicamente activo sobre la percepción del estado de salud y el síndrome de burnout en el profesorado. Para ello, una muestra de 65 docentes que fueron distribuidos en tres grupos según su nivel de actividad física (alta, moderada y baja) correspondiente al Cuestionario Internacional de Actividad Física (IPAQ), cumplimentaron el General Health Questionnaire (GHQ-28) y el Maslach Burnout Inventory (MBI). Los resultados confirman que la actividad física tiene efectos positivos en las dimensiones que definen la percepción de la salud y el burnout; no obstante, en algunas de las dimensiones se obtienen diferencias significativas cuando la intensidad de actividad física es moderada.

PALABRAS CLAVE: Actividad Física, Salud, Docentes, Burnout. 


\begin{abstract}
Teaching can lead to both physical and emotional exhaustion and this can have serious repercussions on a person's health. This study examines the impacts of being physically active on a teacher's perception of health state and burnout syndrome. After stratifying the teachers enrolled $(n=65)$ by physical activity level (high, moderate or low) based on the International Physical Activity Questionnaire (IPAQ), they completed the General Health Questionnaire (GHQ28) and the Maslach Burnout Inventory (MBI). Results indicate that moderate and high levels of physical activity have beneficial effects on the dimensions that define the perception of health and burnout. However, in the dimension social dysfunction, significant impacts emerged only when the intensity of physical activity was moderate.
\end{abstract}

KEY WORDS: Physical Activity, Health, Teachers, Burnout

\title{
INTRODUCCIÓN
}

La actividad docente supone un proceso de interacción con el alumnado y con las familias, en un contexto específico como es el aula de un centro escolar, donde las condiciones retributivas, materiales y de recursos humanos vienen reguladas por modelos de organización y de gestión instaurados en un marco laboral. Las exigencias laborales que se desprenden del desempeño profesional tienen incidencia en el estado de salud y el bienestar de los docentes, y pueden tener consecuencias a nivel físico como el aumento de la presión arterial, problemas gastrointestinales o afecciones musculo-esqueléticas (Bermúdez, Martínez, Rius y Esteve, 2004; Erick y Smith, 2011), y a nivel psicológico causando estrés, ansiedad o fatiga general (Moyano y Riaño-Hernández, 2013; Sándström, Rhodin, Lundberg, Olsson y Nyberg, 2005; Sangganjanavanich y Balkin, 2013). Numerosos estudios han puesto de relieve los problemas de salud que están asociados al profesional docente (Aluja, 1997; Cropley, Steptoe y Joekes, 1999; Chan, Lai, Ko y Boey, 2000; Jamal, 1999; Kinnunen y Salo, 1994; Leung, Siu y Spector, 2000).

El profesorado representa un grupo con alto riesgo de padecer burnout (Schamer y Jackson, 1996; Serrano, Moya-Albiol y Salvador, 2008), lo que repercute en la calidad de vida de los docentes (Schwab, Jackson y Schuller, 1986; Yang et al., 2009) y un deterioro progresivo de su función profesional, y representa una disfunción personal y profesional en un contexto laboral de tipo asistencial. Las consecuencias del burnout se transfieren al desarrollo profesional, donde existe una mayor frecuencia de ausencias del puesto de trabajo, falta de compromiso con la labor docente, impuntualidad e incluso el abandono (Bardo, 1979; Moriana y Herruzco, 2004). En uno de los primeros estudios donde se analizan las causas que subyacen a las bajas laborales de profesores de enseñanza, Chakravorty (1989) concluye que un $77 \%$ de las bajas de larga duración se debían a patologías de tipo psicológico como la depresión, la ansiedad y el estrés. 
El modelo multidimensional del burnout (Maslach y Leiter, 1999) incluye la experiencia de estrés, la evaluación de los otros y la evaluación de uno mismo, que conforman un proceso que se inicia con el cansancio emocional donde el docente es incapaz de mantener recursos emocionales necesarios para afrontar las demandas laborales, dando paso después a la despersonalización donde surgen sentimientos negativos de distanciamiento con respecto a los alumnos, y confluye en una carencia de realización personal, con sentimientos de insatisfacción con el desempeño profesional (Dorman, 2003).

En la valoración de la salud encontramos diversidad de metodologías en función de la perspectiva objetiva o subjetiva que se considere, y también de aquellos indicadores internos o externos que se tomen de referencia. Destaca el grado de funcionalidad, que incide sobre las capacidades físicas para acometer las tareas diarias y para satisfacer los cometidos profesionales y las funciones sociales. Este proceso en la vida humana puede verse interrumpido, de forma más o menos transitoria, por alguna circunstancia que pueda acaecer a lo largo de la vida, y que pueda afectar a la percepción del individuo sobre su salud. Los cuestionarios de salud percibida contienen indicadores de bienestar y de sintomatología, que suponen una valoración global del estado de salud (Robine, Jagger y Egidi, 2000).

La condición física de las personas influye en la flexibilidad, fuerza, coordinación y equilibrio, lo que propicia una mayor estabilidad postural, reduciendo el riesgo de caídas y fracturas. Además, la actividad física mejora la autoestima, retrasa el deterioro cognitivo, alivia los síntomas de depresión y posibilita la integración social (Nelson et al., 2007), demorando el envejecimiento y aumentando el periodo productivo de la vida.

El objetivo de este estudio consiste en analizar los efectos que pueden derivarse de ser una persona físicamente activa en la salud percibida de los docentes y su incidencia sobre los componentes que definen el síndrome de burnout.

\section{MÉTODO}

\section{PARTICIPANTES}

Para este estudio se ha contado con una muestra de 65 profesores cuya participación ha sido voluntaria. De ellos, el $52,31 \%(N=34)$ son hombres, y el $47,69 \%(N=31)$ mujeres. Las edades oscilan entre los 25 y los 62 años $(M=$ 44,80 ; DT $=9,23$ ). Como criterio de inclusión en la muestra, los profesores debían estar en activo en cualquiera de los niveles de enseñanza infantil, primaria o secundaria, siendo seleccionados por muestreo no probabilístico de tipo causal de acuerdo a la facilidad de acceso a los participantes. La muestra se dividió en tres grupos, alta participación, moderada participación y baja participación, en función del volumen de actividad física que realizaban en sus tareas cotidianas y específicas de ejercicio físico, que fue calculado en los participantes según el análisis del Cuestionario Internacional de Actividad Física (IPAQ). 


\section{INSTRUMENTOS}

En este estudio se utilizó el Cuestionario Internacional de Actividad Física (IPAQ) elaborado por el International Consensus Group, y traducido al español por Román, Ribas, Ngo, y Serra (2013), que mide el patrón de actividad física a través de preguntas en todas las áreas de la vida diaria y obteniendo como resultado una mediana en MET-minutos. Establece tres categorías (Craig et al., 2003): alta participación (sujetos que realizan actividades semanales de intensidad vigorosa al menos 3 días sumando un mínimo total de al menos 1500 MET-minutos/semana, o cualquier combinación de actividad física de al menos 3000 MET-minutos/semana), moderada participación (sujetos que realizan 3 o más días semanales de actividad física vigorosa de al menos 20 minutos por día, o 5 o más días de intensidad física moderada al menos 30 minutos por día, o cualquier combinación de actividad física de al menos 600 METminutos/semana) y baja participación (sujetos que no realizan actividad física o ésta es escasa).

Para determinar la percepción del estado de salud de los docentes, se empleó el General Health Questionnaire (GHQ-28) de Goldberg y Hillier (1979), en su versión de 28 ítems. Consta de 4 escalas formadas cada una por 7 ítems con respuesta tipo Likert que evalúan las dimensiones de síntomas somáticos, ansiedad e insomnio, disfunción social y depresión grave. En el análisis de la consistencia interna de la prueba, se obtuvo un alfa de Cronbach de 0,$94 ; y$ en los factores oscilaron entre 0,77 y 0,93 .

Para la evaluación del burnout, se utilizó el Maslach Burnout Inventory (MBI) de Maslach y Jackson (1986), en la versión traducida al español por Seisdedos (1997), que consta de 22 ítems referidos a actitudes, emociones y sentimientos que el docente muestra hacia el trabajo y hacia el alumnado, y que analizan tres dimensiones: cansancio emocional, despersonalización y realización personal. Las respuestas son tipo Likert de 0 a 6 , donde 0 significa que lo expuesto no sucede nunca y 6 que sucede todos los días. En la consistencia interna, se encontraron alfas de Cronbach que oscilaron entre 0,79 y 0,86 .

\section{PROCEDIMIENTO}

La metodología empleada en esta investigación es transversal. Se contactaron con los docentes de forma presencial explicándoles el propósito del estudio y requiriendo su participación voluntaria. Fueron convocados individualmente para que cumplimentaran los cuestionarios en un espacio de su centro escolar donde hubiera un ambiente tranquilo y en un horario que permitiera la realización de las pruebas en el tiempo necesario. Se recabó el consentimiento informado de todos los participantes, asegurando además la confidencialidad de los datos ya que los cuestionarios eran anónimos.

\section{ANÁLISIS ESTADÍSTICO}


Inicialmente se aplicó la prueba de Shapiro-Wilk para comprobar la normalidad de las distintas variables, cumpliéndose en todas las variables los criterios de normalidad. Posteriormente, se realizó un ANOVA de un factor, tras comprobar que existía homocedasticidad mediante el estadístico de Levene, aplicándose un test post-hoc de Scheffé en aquellas variables en las que se habían encontrado diferencias estadísticamente significativas entre grupos. El nivel de significación estadística fue establecido en $p<0,05$.

Todo el análisis estadístico se realizó en el paquete estadístico SPSS para Windows (versión 17.0).

\section{RESULTADOS}

El análisis de la actividad física de los sujetos de la muestra mediante el IPAQ estableció que el grupo de alta actividad física estuvo conformado por 23 docentes, mientras que el de moderado nivel de actividad lo constituyeron 17 docentes y 25 el de bajo nivel de actividad física. La distribución de los participantes en el estudio en función del nivel de actividad física que realizan, aparece en la Tabla 1.

Tabla 1. Distribución de los participantes en grupos según el nivel de actividad física

\begin{tabular}{cccccccc}
\hline \multicolumn{2}{c}{$\begin{array}{c}\text { Alta Actividad } \\
\text { Física }\end{array}$} & \multicolumn{2}{c}{$\begin{array}{c}\text { Moderada Actividad } \\
\text { Física }\end{array}$} & \multicolumn{2}{c}{$\begin{array}{c}\text { Baja Actividad } \\
\text { Física }\end{array}$} & \multicolumn{2}{c}{ Total } \\
\hline $\mathrm{n}$ & $\%$ & $\mathrm{n}$ & $\%$ & $\mathrm{n}$ & $\%$ & $\mathrm{n}$ & $\%$ \\
\hline 23 & $35,38 \%$ & 17 & $26,16 \%$ & 25 & $38,46 \%$ & 65 & $100 \%$ \\
\hline
\end{tabular}

En la Tabla 2 se muestra el análisis del efecto de los distintos niveles de actividad física con las dimensiones de los cuestionarios GHQ-28 y MBI, comprobándose que existe diferencias estadísticamente diferentes entre los distintos grupos de nivel de actividad física en todas las dimensiones de ambos cuestionarios $(p<0,05)$.

Tabla 2. Puntuaciones en los cuestionarios GHQ-28 y MBI en función del nivel de actividad física. Valores presentados como media \pm desviación típica

\begin{tabular}{ccccccc}
\hline \multicolumn{2}{c}{ Variable } & Alta & Moderada & Baja & $\boldsymbol{F}$ & $\boldsymbol{p}$ \\
\hline GHQ- & Síntoma S. & $3,35 \pm 2,12$ & $5,29 \pm 3,53$ & $5,56 \pm 3,64$ & 3,440 & $0,042^{*}$ \\
28 & Ansiedad & $3,52 \pm 2,43^{\mathrm{a}}$ & $3,76 \pm 2,54$ & $6,44 \pm 5,46$ & 4,001 & $0,023^{*}$ \\
& Disf. Soc. & $6,39 \pm 1,30$ & $6,18 \pm 1,18^{\mathrm{b}}$ & $8,16 \pm 3,60$ & 4,481 & $0,150^{*}$ \\
& Depresión & $0,74 \pm 1,89$ & $0,29 \pm 0,98$ & $3,16 \pm 4,10^{\mathrm{c}}$ & 6,653 & $0,002^{*}$ \\
& GHQ T & $14,00 \pm$ & $15,53 \pm 6,48$ & $23,32 \pm 14,28$ & 5,939 & $0,004^{*}$ \\
\hline $\mathrm{MBI}$ & Cans. Em. & $15,18^{\mathrm{a}}$ & & & & \\
& & $7,35 \pm 8^{\mathrm{a}}$ & $17,94 \pm 8,79$ & $25,36 \pm 11,92$ & 6,597 & $0,003^{*}$ \\
& Despers. & $2,78 \pm 3,50$ & $2,53 \pm 4,51$ & $8,24 \pm 6,45^{\mathrm{c}}$ & 9,274 & $0,000^{*}$ \\
& Real. Pers. & $40,83 \pm 4,26$ & $41,41 \pm 5,32$ & $34,84 \pm 9,80^{\mathrm{c}}$ & 5,825 & $0,005^{*}$ \\
\hline
\end{tabular}

GHQ-28: Health Questionnaire; MBI: Maslach Burnout Inventory

* Diferencias estadísticamente significativas para el factor grupo $(p<0,05)$; ${ }^{\text {a }}$ Diferencias estadísticamente significativos mediante post-hoc de Scheffé entre nivel de actividad física alta y baja; ${ }^{b}$ Diferencias estadísticamente significativos mediante post-hoc de Scheffé entre nivel de actividad física moderada y baja; ${ }^{\circ}$ Diferencias estadísticamente significativos mediante posthoc de Scheffé entre nivel de actividad física alta y moderada con respecto a baja. 
En el cuestionario GHQ-28, el análisis post-hoc de Scheffé reflejó que el nivel de alta actividad física dio lugar a una puntuación significativamente inferior en la puntuación total del GHQ $(14,00 \pm 5,18$ vs $23,32 \pm 14,28, p=0,008)$ (Figura 1), así como en la dimensión ansiedad-insomnio $(3,52 \pm 2,43$ vs 6,44 $\pm 5,46, p=0,042)$. Por su parte, tanto el nivel de alta y moderada actividad física fueron eficaces en la reducción de la dimensión depresión $(0,74 \pm 1,89$ vs $3,16 \pm 4,10, p=0,017$ y $0,29 \pm 0,98$ vs $3,16 \pm 4,10, p=0,008)$. En cuanto a la dimensión disfunción social, sin embargo, únicamente hubo interacción en el nivel de actividad física moderada con respecto al de baja actividad $(6,18 \pm 1,18$ vs $8,16 \pm 3,60, p=0,043)$.

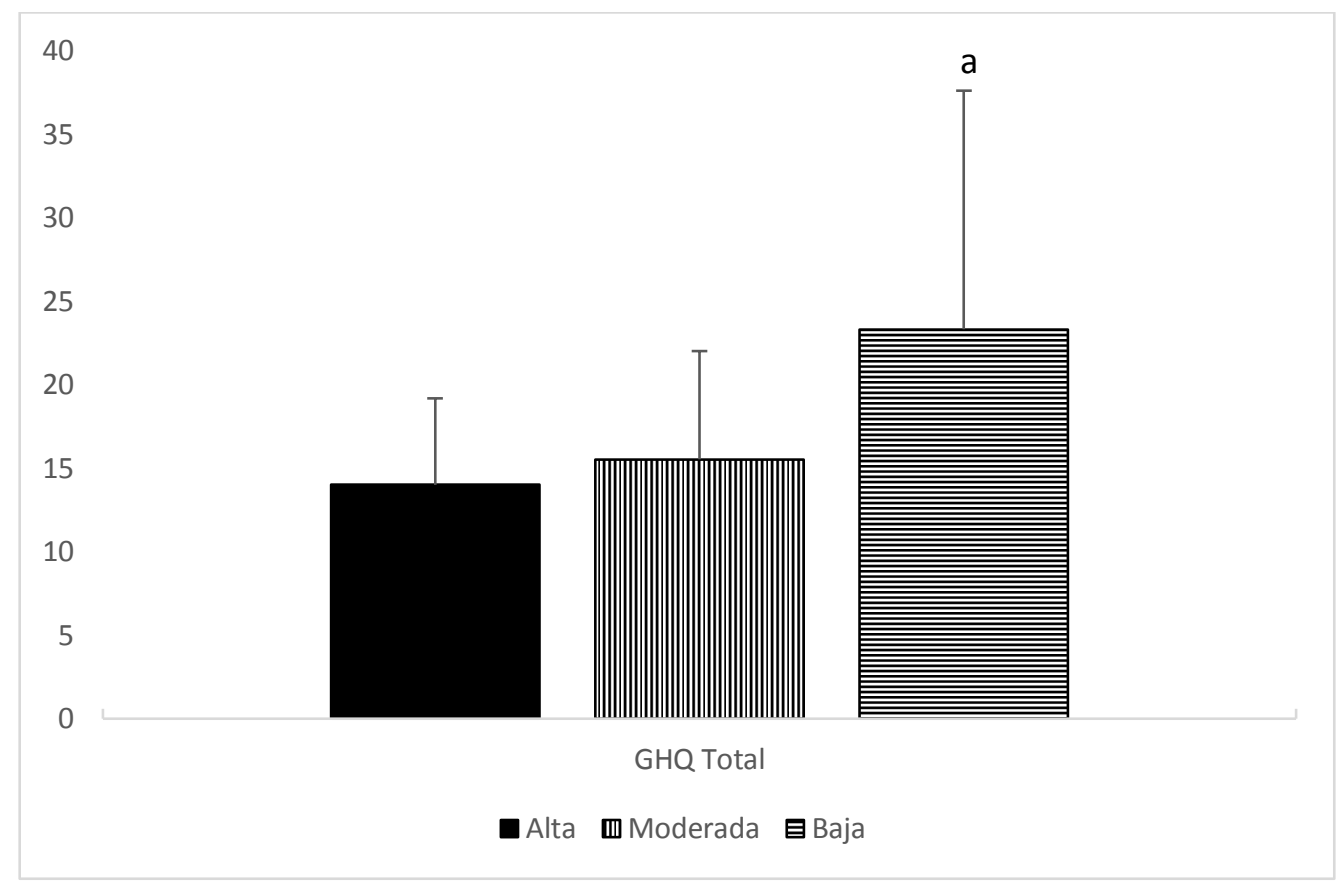

Figura 1. Puntuaciones medias + desviación típica en la puntuación total en el test GHQ a Diferencias estadísticamente significativas entre alta actividad vs baja actividad física $(p<0,05)$ 


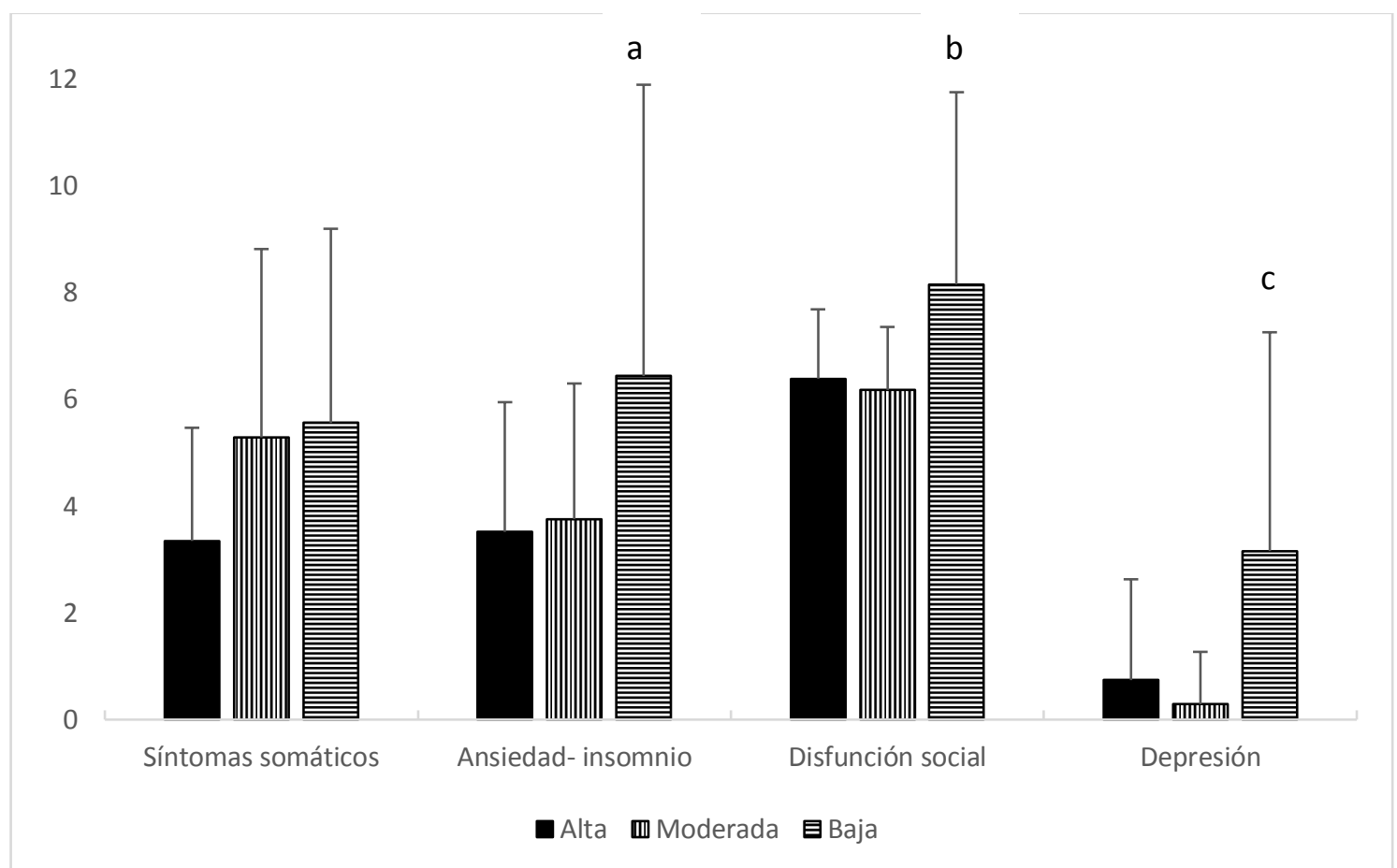

Figura 2. Puntuaciones medias + desviación típica en la puntuación de las distintas dimensiones del test GHQ

${ }^{a}$ Diferencias estadísticamente significativas entre alta actividad vs baja actividad física $(p<0.05)$

${ }^{\mathrm{b}}$ Diferencias estadísticamente significativas entre moderada actividad vs baja actividad física $(p<0,05)$

${ }^{c}$ Diferencias estadísticamente significativas entre alta y moderada actividad vs baja actividad física $(p<0,05)$

En el cuestionario MBI el análisis post-hoc de Scheffé reflejó que el nivel de alta actividad física dio lugar a una puntuación significativamente inferior en la dimensión cansancio emocional $(15,35 \pm 7,98$ vs $25,36 \pm 11,92, p=0,004)$, mientras que tanto el nivel de alta y moderada actividad física fueron eficaces en la reducción de las dimensiones despersonalización $(2,78 \pm 3,50$ vs $8,24 \pm 6,45$, $p=0,002$ y $2,53 \pm 4,51$ vs $8,24 \pm 6,45, p=0,003)$ y un aumento de la realización social $(40,83 \pm 4,26$ vs $34,84 \pm 9,80, p=0,020$ y $0,29 \pm 0,98$ vs $34,84 \pm 9,80$, $p=0,019$ ). 


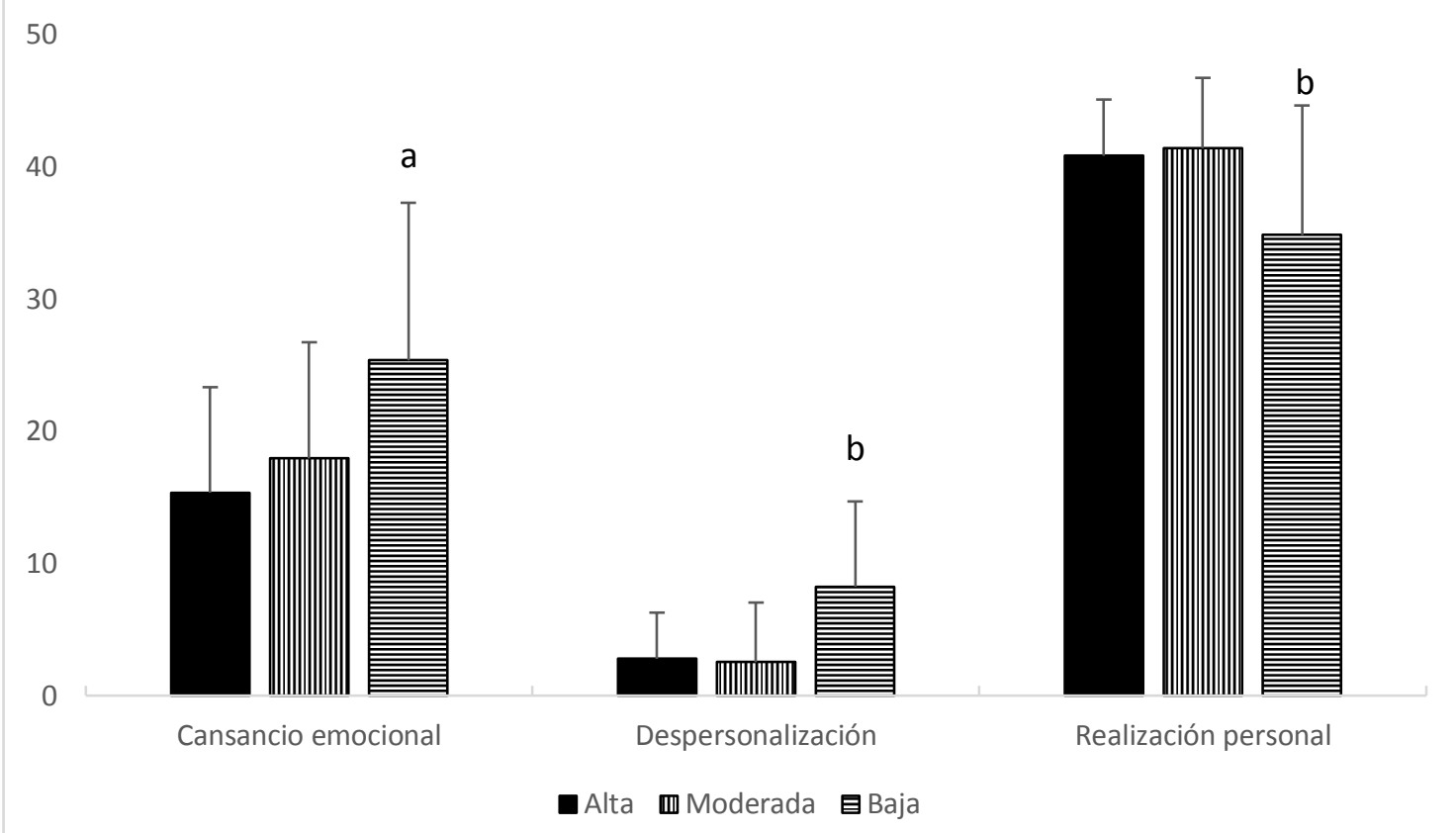

Figura 3. Puntuaciones medias + desviación típica en la puntuación de las distintas dimensiones del test MBI

a Diferencias estadísticamente significativas entre alta actividad vs baja actividad física $(p<0,05)$

${ }^{\mathrm{b}}$ Diferencias estadísticamente significativas entre alta y moderada actividad vs baja actividad física $(p<0,05)$

\section{DISCUSIÓN}

Los resultados encontrados en este trabajo muestran que los profesores que se mantienen físicamente activos presentan una percepción más positiva de su estado de salud que aquellos docentes que tienen un estilo de vida con una baja actividad física. En las dimensiones que definen la salud subjetiva, se han hallado diferencias significativas entre los grupos de profesores que realizan una actividad física alta o moderada, y el grupo de docentes físicamente sedentarios o de baja actividad, obteniendo en todos los factores puntuaciones que definen un mejor estado de salud cuando se realiza actividad física. Por lo tanto, podemos afirmar, al igual que lo hacen otros estudios (De Miguel, Schweiger, Mozas y Hernández, 2011; Pérez y Devís, 2003; Rodríguez, Márquez y De Abajo, 2006; Toker y Biron, 2012), que la actividad física realizada de forma regular, produce beneficios físicos y psicológicos en las profesionales que se dedican a la docencia, disminuyendo los niveles de percepción en los factores que denotan disfunciones o patologías en el estado de salud subjetivo. Estos aspectos suponen mecanismos de protección ante patologías como la ansiedad, cuya diferencia es significativa entre docentes físicamente activos y no activos (McAuley, Márquez, Jerome, Blissmer y Katula, 2002); como la depresión, con diferencias significativas entre el grupo de docentes que tiene alta o moderada actividad física donde el nivel de depresión es bajo respecto de los docentes sedentarios (Anderson et al., 2009); o como la disfunción social, con diferencias significativas entre los docentes que tienen una moderada actividad física y los que son sedentarios, favoreciendo el bienestar y la calidad de vida de las personas (Infante, Goñi y Villarroel, 2011; Netz, Wu, Becker y Tenenbaum, 2005; Pino y Pria-Bankoff, 2007). 
En cuanto al efecto que tiene la actividad física sobre los factores que definen el síndrome de burnout de los docentes, encontramos que, al igual que en otros estudios (Kotova, Rozanov, Alexandrov, e Ivanova, 2017; Papastylianou, Kaila y Polychronopoulos, 2009; Sane, Devin, Jafari y Zohoorian, 2012), existe una relación inversa entre la práctica de actividad física y los niveles de cansancio emocional y despersonalización, y una relación directa con los valores de realización personal; en concreto, se constata que cuanto más activo físicamente sea el docente menor nivel de cansancio emocional percibe, y que una actividad física moderada produce efectos más positivos en la sensación de despersonalización y de autorrealización. Por lo tanto, podemos afirmar que, mantenerse físicamente activo, con una intensidad adecuada, reduce el estado de burnout en los docentes, incrementando la percepción de salud física y mental (García, Carbonell y Delgado, 2010; Oña, 2002). El cansancio emocional impide el desarrollo positivo de la función docente, mostrando una menor implicación y una sensación de agotamiento, mientras que los niveles altos en el factor de despersonalización sugieren una tendencia al distanciamiento profesional respecto del alumnado, revelando una actitud poco sensible a la problemática escolar y a cualquier dificultad que pudiera surgir.

La vida laboral ocupa una gran parte del tiempo diario de las personas, por lo que combatir el sedentarismo, tanto con la actividad habitual en los diferentes momentos del trabajo, del transporte o de las rutinas cotidianas, como con la práctica específica de ejercicio, supone un reto en el desarrollo del bienestar físico y psicológico de los trabajadores (Rosales et al., 2017; Tu, Stump y Clar, 2004). Es preciso que en la función docente se incorporen tanto estrategias de intervención externas, relacionadas con la organización del sistema educativo y la gestión de los centros docentes, como estrategias personales que supongan factores de protección que compensen el agotamiento físico y el desgaste emocional. Para ello, el docente debe tomar conciencia de la importancia de mantenerse físicamente activo como estilo de vida relacionado con la salud, y la necesidad de establecer intensidades en la actividad física adecuadas al desarrollo de sus rutinas diarias tanto del ejercicio profesional como de la vida personal, familiar y social.

\section{REFERENCIAS BIBLIOGRÁFICAS}

Aluja, A. (1997). Burnout profesional en maestros y su relación con indicadores de salud mental. Boletín de Psicología, 55, 47-61.

Anderson, L.M., Quinn, T.A., Glanz, K., Ramírez, G., Kahwati, L.C., Johnson, D.B., Buchanan, L.R., Archer, W., Chattopadhyay, S., Kalra, G.P., y Katz, D.L. (2009). The effectiveness of worksite nutrition and physical activity interventions for controlling employee overweight and obesity. American Journal of Preventive Medicine, 37(4), 340-357. Doi:10.1016/j.amepre.2009.07.003.

Bardo, P. (1979). The Pain of Teacher Burnout: A case History. Phi Delta Kappan, 61(4), 252-254. 
Bermúdez, R., Martínez, G., Rius, F., y Esteve, J.M. (2004). Relaciones entre el uso de la voz y el burnout en los docentes de Educación Infantil y Primaria de Málaga. Revista Española de Pedagogía, 227, 85-102.

Chakravorty, B. (1989). Mental health among school teachers. En M. Cole y S. Walker (Eds.), Teaching and Stress (pp. 69-82). Philadelphia: Open University Press.

Chan, K.B., Lai, G., Ko, Y.C., y Boey, K.W. (2000). Work stress among six professional groups: The Singapore experience. Social Science \& Medicine, 50(10), 1415-1432. Doi:10.1016/s0277-9536(99)00397-4.

Craig, C.L., Marshall, A.L., Sjöström, M., Bauman, A.E., Booth, M.L., Ainsworth, B.E., Pratt, M., Ekelund, U. Yngve, A., Sallis, J.F., y Oja, P. (2003). International physical activity questionnaire: 12-country reliability and validity. Medicine and Science in Sports and Exercise, 35(8), 1381-1395. Doi:10.1249/01.MSS.0000078924.61453.FB.

Cropley, M., Steptoe, A., y Joekes, K. (1999). Job strain and psychiatric morbidity. Psychological Medicine, 29(6), 1411-1416. https://doi.org/10.1017/S003329179900121X.

De Miguel, J.M., Schweiger, I., Mozas, O.d.I., y Hernández, J.M. (2011). Efecto del ejercicio físico en la productividad laboral y el bienestar. Revista de Psicología del Deporte, 20(2), 589-604.

Dorman, J.P. (2003). Relationship between school and classroom environment and teacher burnout: A LISREL analysis. Social Psychology of Education, 6(2), 107-127. https://doi.org/10.1023/A:1023296126723.

Erick, P., y Smith, D.R. (2011). A systematic review of muskoloskeletal disorders among school teachers. BCM Muskoloskeletal Disorders, 12, 260-271. Doi:10.1186/1471-2474-12-260.

García, A., Carbonell, A., y Delgado, M. (2010). Beneficios de la actividad física en personas mayores. Revista Internacional de Medicina y Ciencias de la Actividad Física y el Deporte, 10(40), 556-576.

Goldberg, D.P., y Hillier, V.F. (1979). A scaled version of the General Health Questionnaire. Psychological Medicine, 9(1), 139-145.

Infante, G., Goñi, A., y Villarroel, J. (2011). Actividad física y autoconcepto, físico y general, a lo largo de la edad adulta. Revista de Psicología del Deporte, 20(2), 429-444.

Jamal, M. (1999). Job stress and employee well-being: a cross-cultural empirical study. Stress Medicine, 15(3), 153-158.

Kinnunen, U., y Salo, K. (1994). Teacher stress: an eight-year follow-up study on teachers' work, stress, and health. Anxiety, Stress, and Coping, 7, 319337.

Kotova, M.B., Rozanov, V.B., Alexandrov, A.A., e Ivanova, E.I. (2017). Professional burnout and quality of life in teachers. Voprosy Psikhologii, 2, 67-79.

Leung, T., Siu, O., y Spector, P.E. (2000). Faculty stressors, job satisfaction, and psychological distress among university teachers in Hong Kong: the role of locus of control. International Journal of Stress Management, 7(2), 121138. https://doi.org/10.1023/A:1009584202196.

Maslach, C., y Jackson, S.E. (1986). Maslach Burnout Inventory (2ª ed, $19811^{1^{\underline{a}}}$ ed.). Palo Alto, California: Consulting Psychologists Press. 
Maslach, C., y Leiter, M.P. (1999). Teacher burnout: A research agenda. En R. Vandenberghe y A.M. Huberman (Eds.), Understanding and preventing teacher burnout (pp. 295-303). New York: Cambridge University Press.

McAuley, E., Márquez, D.X., Jerome, G.J., Blissmer, B., y Katula, J. (2002). Physical activity and physique anxiety in older adults: fitness and efficacy influences. Aging and Mental Health, 6(3), 220-230. Doi:10.1080/13607860220142459.

Moriana, J.A., y Herruzco, J. (2004). Estrés y burnout en profesores. International Journal of Clinical and Health Psychology, 4(3), 597-621.

Moyano, N., y Riaño-Hernández, D. (2013). Burnout escolar en adolescentes españoles: Adaptación y validación del School Burnout Inventory. Ansiedad y Estrés, 2013, 19(1), 95-113.

Nelson, M.E., Rejeski, W.J., Blair, S.N., Duncan, P.W., Judge, J.O., King, A.C., Macera, C.A., y Castaneda, C. (2007). Physical activity and public health in older adults: Recommendation from the American College of Sports Medicine and the American Heart Association. Medicine and Science in Sports and Exercise, 39(8), 1435-1445. Doi:10.1249/mss.0b013e3180616aa2.

Netz, Y., Wu, M.J., Becker, B.J., y Tenenbaum, G. (2005). Physical activity and psychological well-being in advanced age: A meta-analysis of intervention studies. Psychology and Aging, 20(2), 272-284. Doi:10.1037/08827974.20.2.272.

Oña, A. (2002). La ciencia en la actividad física: Viejos y nuevos problemas. Revista Motricidad, 9, 9-42.

Papastylianou, A., Kaila, M., y Polychronopoulos, M. (2009). Teachers'burnout, depression, role ambiguity and conflict. Social Psychology of Education, 12(3), 295-314. https://doi.org/10.1007/s11218-008-9086-7.

Pérez, V., y Devís, J. (2003). La promoción de la actividad física relacionada con la salud. La perspectiva de proceso y de resultado. Revista Internacional de Medicina y Ciencias de la Actividad Física y del Deporte, 3(10), 69-74.

Pino, C., y Pria-Bankoff, A.D. (2007). Estudio comparativo de nivel de capacidad física, estrés laboral y molestia musculo esqueléticas en trabajadores. Efdeportes, 115. Recuperado en http://www.efdeportes.com.

Robine, J.M., Jagger, C., y Egidi, V. (2000). Selection of a coherent set of health indicators. Final draft. A first step towards a user's guide to health experiences for the European Union.Euro-REVES (France).

Rodríguez, J., Márquez, S., y De Abajo, S. (2006). Sedentarismo y salud: Efectos beneficiosos de la actividad física. Apunts: Educación Física y Deportes, 83, $12-24$.

Román, B., Ribas, L., Ngo, J., y Serra, L. (2013). Validación en población catalana del cuestionario internacional de actividad física. Gaceta Sanitaria, 27(3), 254-257. Doi:10.1016/j.gaceta.2012.05.013.

Rosales, Y., Orozco, D., Yaulema, L., Parreño, A., Caiza, V., Barragán, V., Ríos, A., y Peralta, L. (2017). Actividad física y salud en docentes. Una revisión. Apunts, 52(196), 159-166.

Sándström, A., Rhodin, I.N., Lundberg, M., Olsson, T., y Nyberg, L. (2005). Impaired cognitive performance in patients with chronic burnout syndrome. Biological Psychology, 69(3), 271-279. Doi:10.1016/j.biopsycho.2004.08.003. 
Sane, M.A., Devin, H.F., Jafari, R., y Zohoorian, Z. (2012). Relationship between physical activity and it's components with burnout in academic members of Daregaz Universities. Procedia - Social and Behavioral Sciences, 46, 4291-4294. Doi:10.1016/j.sbspro.2012.06.242.

Sangganjanavanich, V.F., y Balkin, R.S. (2013). Burnout and job satisfaction among counselor educators. Journal of Humanistic Counseling, 52(1), 6779. https://doi.org/10.1002/j.2161-1939.2013.00033.x.

Schamer, L.A., y Jackson, M. (1996). Coping with stress: Common sense about teacher burnout. Education Canada, 36(2), 28-31.

Schwab, R.L., Jackson, S.E., y Schuler, R.S. (1986). Educator burnout sources and consequences. Educator Research Quarterly, 10(3), 15-30.

Seisdedos, N. (1997). Manual MBI, Inventario "Burnout" de Maslach. Madrid: TEA Ediciones.

Serrano, M.A., Moya-Albiol, L., y Salvador, A. (2008). The role of gender in teachers perceived stress and heart rate. Journal of Psychophysiology, 22(1), 58-64. https://doi.org/10.1027/0269-8803.22.1.58.

Toker, S., y Biron, M. (2012). Job burnout and depression: Unraveling their temporal relationship and considering the role of physical activity. Journal of Applied Psychology, 97(3), 699-710. Doi:10.1037/a0026914.

Tu, W., Stump, T., y Clar, D. (2004). The effects of health and environment on exercise-class participation in older, urban women. Journal of Aging and Physical Activity, 12(4), 480-496. Doi:10.1123/japa.12.4.480.

Yang, X., Ge, C., Hu, B., Chi, T., y Wang, L. (2009). Relationship between quality of life and occupational stress among teachers. Public Health, 123(11), 750-755. Doi:10.1016/j.puhe.2009.09.018.

Número de citas totales / Total references: 43 (100\%)

Número de citas propias de la revista / Journal's ownreferences: 2 (4,6\%)

Rev.int.med.cienc.act.fís.deporte- vol. X - número X - ISSN: 1577-0354 